\title{
COMÉRCIO E INTEGRAÇÃO DOS ESTADOS BRASILEIROS
}

\author{
Fernanda Maria de Almeida ${ }^{l}$ \\ Orlando Monteiro da Silva
}

\begin{abstract}
Resumo - Como no Brasil há significativa assimetria no volume de produção e comércio entre os estados, este estudo objetivou mensurar o efeito-fronteira no comércio intranacional e internacional do país, utilizando-o com indicador do grau de integração. Tal efeito mede o viés para o comércio interno de um país ou estado, em comparação com o comércio externo. O modelo de gravidade, com a inclusão de diferentes variáveis dummies, foi utilizado, como referencial analítico, neste estudo. Os resultados mostraram que o comércio intra-estadual no Brasil variava de 32 a 96 vezes mais do que o interestadual e internacional, respectivamente, e o interestadual, em torno de 34 vezes maior que o internacional. O efeito-fronteira, calculado para cada estado, sobre o comércio com os demais estados e com o resto do mundo apresentou valores menores e maior integração comercial nos estados das regiões Sudeste e Sul do Brasil.
\end{abstract}

Palavras-chave: Comércio internacional, efeito-fronteira, modelo de gravidade.

\section{Introdução}

As relações de comércio dos países são favorecidas pelo surgimento de novas tecnologias no processo produtivo, pela especialização da mãode-obra e pela ampliação e desenvolvimento dos meios de comunicação e de transporte. Além disso, maior mobilidade financeira e redução de barreiras comerciais entre os países e blocos regionais contribuem para a integração dos mercados no comércio internacional.

A identificação de fatores que favoreçam as relações comerciais permite a mensuração do grau de integração dos mercados, dos custos das transações comerciais, dos ganhos potenciais do comércio e, ainda, dos desvios ou preferências do comércio intranacional em relação ao

\footnotetext{
Bolsista da Fapemig. E-mail: f_m_almeida@yahoo.com.br

2 Professor do Departamento de Economia da Universidade Federal de Viçosa. E-mail: odasilva@ufv.br
} 
internacional. Tais preferências pelo comércio interno são comumente conhecidas como efeito-fronteira. Assim, quando um país comercializa mais internamente do que com os demais países, diz-se que o comércio é afetado pelo efeito-fronteira, o que leva o país a apresentar baixo nível de integração.

McCallum (1995) e Helliwell (1996) foram um dos primeiros autores a realizar estudos sobre o efeito-fronteira, utilizando o chamado modelo de gravidade para comparar o comércio entre as províncias do Canadá e dos Estados Unidos. Em sua forma básica, tal modelo explica as relações bilaterais de comércio por meio de variáveis como distância física e tamanho econômico dos países ou estados. Esses autores estimaram o efeito-fronteira pela inclusão de uma variável dummy no modelo gravitacional, tendo como base o comércio intra-província. Os resultados obtidos por McCallum mostraram que o comércio entre duas províncias canadenses era cerca de 20 vezes maior que o dessas províncias com os Estados Unidos, o que indica a existência de um viés nas compras domésticas comparadas às internacionais, nas províncias canadenses.

No geral, países em desenvolvimento possuem estreitas relações de comércio internacional. No Brasil, por exemplo, as exportações são voltadas principalmente para produtos que exigem mão-de-obra intensiva, como os agrícolas e as commodities. Quanto às importações, grande parte delas é de produtos ou de fatores nos quais se empregam tecnologias avançadas, uma vez que as vantagens comparativas no desenvolvimento de novos tipos desses produtos ainda são pequenas.

O Brasil ainda apresenta distribuição econômica muito heterogênea entre seus estados, no que tange ao volume de produção e comércio. Estados como São Paulo, Rio de Janeiro e Minas Gerais são responsáveis pela maior parte do volume de comércio e da produção do país, enquanto os das regiões Norte e Nordeste apontam baixos níveis de produção e comércio. São necessários, portanto, estudos que auxiliem no entendimento e na explicação das causas dessa assimetria, para que medidas políticas possam ser tomadas para sua correção. Assim, este estudo objetivou 
mensurar o efeito-fronteira que há no comércio entre os estados brasileiros (intranacional) e aquele entre os estados e o mercado externo (internacional) e, desse modo, indicar um grau de integração do mercado doméstico.

\section{Metodologia}

O uso do modelo de gravidade para explicar as relações econômicas e comerciais foi inicialmente proposto por Jan Tinbergen, em 1962. Segundo esse autor, as relações bilaterais de comércio eram determinadas pelo tamanho econômico de cada parceiro comercial, usualmente representado pelos Produtos Internos Bruto (PIBs) e pela distância física entre eles. O tamanho da massa econômica de cada país (PIB) é um fator favorável ao volume de transações, uma vez que países desenvolvidos tendem a realizar maior volume de comércio. A variável distância pode ser considerada uma proxy para os custos do comércio. Tal variável representa uma resistência ao fluxo comercial, em razão dos elementos de natureza econômica,como os custos e o tempo do transporte e, ainda, os custos de informação. Em sua forma original, a equação gravitacional é expressa por:

$\omega_{i j}=\varphi \frac{Y_{i} Y_{j}}{D_{i j}}$

em que o termo $\omega_{i j}$ representa o fluxo de comércio entre dois países, i e $\mathrm{j} ; \varphi$ é uma constante gravitacional; $Y_{i}$ e $Y_{j}$ indicam, respectivamente, o PIB do país exportador (a capacidade de oferta) e do país importador (a capacidade de demanda); e $D_{i j}$ é a distância entre eles. A equação (1) é, usualmente, apresentada na forma log-linear: 
$\ln \omega_{i j}=\delta_{0}+\delta_{1} \ln Y_{i}+\delta_{2} \ln Y_{j}+\delta_{3} \ln D_{i j}+\varepsilon_{i j}$,

em que os $\delta^{\prime} s$ são diretamente as elasticidades de cada variável utilizada e $\varepsilon_{i j}$ indica o termo de erro. Na estimação da equação (2) são esperados sinais positivos para os coeficientes das variáveis PIB e negativo para o coeficiente da variável distância.

Várias modificações foram introduzidas na forma básica do modelo gravitacional da equação (1). Linnemann (1966), por exemplo, citado por Azevedo (2004), introduziu no modelo a variável tamanho da população, como forma de ponderar o coeficiente entre produção para o mercado doméstico e para o mercado externo. No Brasil, diversos autores (Piani e Kume, 2000; Sá Porto, 2002; Azevedo, 2004 e Azevedo et al., 2006) introduziram variáveis dummies para captar os efeitos no comércio da adesão de determinado país aos acordos econômicos regionais. Uma boa resenha sob os fundamentos teóricos do modelo de gravidade pode ser encontrada no trabalho de Morais (2005).

Hidalgo e Vergolino (1997) foram os pioneiros no Brasil na utilização do modelo gravitacional para explicar o comércio regional. Esses autores avaliaram as características do comércio do Nordeste e as das demais regiões do país e de outros países, analisando os impactos das fronteiras diante dos padrões de comércio regional e internacional. Recentemente, Silva et al. (2004), ao atualizarem essa análise, considerando também o comércio intra-estados do Nordeste, encontraram resultados semelhantes.

A equação gravitacional tem sido também utilizada para medir os efeitos nos fluxos de comércio, na renda per capita (Piani e Kume, 2000), na similaridade da renda entre os parceiros comerciais e na adjacência entre eles (Silva et al. 2007).

Daumal e Zignago (2005) empregaram o modelo de gravidade para estimar o grau de integração entre os estados brasileiros, no período 1991/99, considerando 164 países estrangeiros na amostra, em que mais da metade 
das observações com fluxos comerciais eram iguais a zero. A variável dependente adotada foi a razão das exportações sobre o produto dos PIBs, e as variáveis explicativas foram a distância e um conjunto de variáveis dummies para medir o efeito-fronteira.

A proposta deste estudo consistiu em medir o efeito-fronteira (preferência pelo comércio local) nos níveis intra e interestados brasileiros, além do nível internacional, utilizando o modelo gravitacional básico, com as exportações como variável dependente e um conjunto de variáveis dummies para captar o efeito da adjacência e do viés doméstico sobre o comércio. Dessa forma, obteve-se, explicitamente, o efeito dos PIBs nas exportações. A equação estimada apresenta a seguinte forma matemática:

$$
\ln \omega_{i j}=\delta_{0}+\delta_{1} \ln Y_{i}+\delta_{2} \ln Y_{i}+\delta_{3} \ln D_{i j}+\sum \phi_{n} B_{i j}+\varepsilon_{i j}
$$

em que

$\omega_{i j}$ são as exportações do estado i para o estado ou país j;

$Y_{i}$ e $Y_{j}$ são os PIBs nominais do estado i e do estado ou país j;

$D_{i j}$ é a distância, em km, do estado i para o estado ou país j;

$B_{i j}$ é um conjunto de variáveis dummies;

$\delta_{1}$ e $\delta_{2}$ são parâmetros que representam as elasticidades de exportação em relação ao PIB do estado i e do estado ou país j;

$\delta_{3}$ é o parâmetro que representa a elasticidade de exportação em relação à distância entre as localidades i e j;

$\phi$ correspondem aos impactos das variáveis dummies utilizadas; e $\varepsilon_{i j}$ indica o termo de erro aleatório. 
O conjunto de variáveis dummies é utilizado para captar a influência da adjacência entre os estados e,ou, países e o viés doméstico do comércio entre os estados e os países estrangeiros, ou seja, o efeito-fronteira.

Os dados sobre o fluxo de comércio interestadual total (exportações) foram obtidos das Matrizes de Comércio Interestadual, elaboradas por Vasconcelos e Oliveira (2006). As exportações estaduais para os principais países importadores foram obtidos no site do Ministério do Desenvolvimento, Indústria e Comércio Exterior (MDIC), e os dados sobre o PIB e a população (nacional e por unidade da federação), do Instituto Brasileiro de Geografia e Estatística (IBGE). Os mesmos dados sobre os países selecionados foram retirados do banco de dados "Penn World Table". Os PIBs dos países foram transformados em reais, utilizando-se a taxa de câmbio média (reais/US\$), para o ano de 1999. As distâncias, medidas em km, foram obtidas do sítio Areaseg.com e representam as distâncias físicas entre as capitais de cada estado. Para os demais países, foi considerada a distância dos estados até Brasília, e de lá até a capital de cada um dos países, sendo os dados obtidos no sítio do Centro de Estudos Prospectivos e de Informações Internacionais (CEPII).

Toda a análise realizada corresponde ao ano de 1999 (ano mais recente para o qual informações sobre o comércio interestadual estão disponíveis), com observações para os 26 estados brasileiros, mais o Distrito Federal, e para os 46 principais países parceiros comerciais do Brasil.

\section{Resultados}

Na Tabela 1 são apresentadas as estimativas do modelo proposto para o comércio agregado entre os estados brasileiros. Os resultados estão apresentados em quatro equações, de modo a expor diferentes especificações do modelo. Os coeficientes estimados são significativos e os sinais, coerentes com o esperado. Os valores de $\mathrm{R}^{2}$ variam de 0,68 a 0,83 , o que indica que as variáveis têm alto poder explicativo sobre as 
exportações estaduais. Todas as equações foram estimadas pelo método dos Mínimos Quadrados Ordinários (MQO), com os resultados já corrigidos para auto-correlação, pelo método de Cochrane-Orcutt (processo auto-regressivo de primeira ordem - AR(1)). Os desvios-padrão, utilizados nos testes de hipóteses, foram obtidos por meio do estimador de White.

Todas as equações foram acrescidas de uma variável dummy, que tinha o valor 1 para os estados e, ou, países adjacentes, e zero, caso contrário, para medir o efeito dessa proximidade nas exportações. Castro et al. (1999), ao estudarem o efeito do custo de transporte no comércio interestadual brasileiro, chamaram atenção para a importância da proximidade dos estados. Segundo esses autores, "há efeitos de vizinhança entre os estados não capturados pelas variáveis tradicionais", por isso, optou-se, aqui, pelo uso da dummy para adjacência.

As equações de (1) a (3) variam também pelo tipo de variável dummy utilizada e por apresentarem, cada uma, 1.361 observações, que equivalem ao comércio intra e entre os estados, e deles com os principais países parceiros comerciais. A equação (4) exclui o comércio dos estados com os países, utilizando, portanto, somente 708 observações.

Na equação (1) introduziu-se uma variável dummy chamada EST, para medir a "preferência" de comércio interno de um estado brasileiro, comparado com os demais estados ou com os parceiros internacionais, que teve o valor 1, para o comércio intra-estadual, e zero, caso contrário. Na equação (2) considerou-se uma variável dummy, chamada BRA, que assumiu o valor 1 para o comércio interestadual e zero, caso contrário. Nas equações (3) e (4) introduziu-se um conjunto de 27 dummies, uma para cada um dos 26 estados, mais o Distrito Federal. A equação (3) avalia o comércio intra-estado na presença das transações internacionais, enquanto a equação (4) mede o quanto os estados comercializam internamente, desconsiderando os parceiros internacionais. 
Tabela 1 - Estimativas do modelo de gravidade para o comércio no Brasil (MQO)

\begin{tabular}{|c|c|c|c|c|c|}
\hline & \multirow{2}{*}{ Parâmetros } & \multicolumn{4}{|c|}{ Equações } \\
\hline & & (1) & (2) & (3) & (4) \\
\hline \multirow{2}{*}{\multicolumn{2}{|c|}{ Constante }} & $-6,282$ & $-13,105$ & $-14,797$ & $-21,407$ \\
\hline & & $(-3,232)^{*}$ & $(-10,408)^{*}$ & $(-11,041)^{*}$ & $(-18,088)^{*}$ \\
\hline \multirow{2}{*}{\multicolumn{2}{|c|}{$\ln Y_{i}$}} & 0,947 & 1,065 & 1,091 & 0,917 \\
\hline & & $(8,520)^{*}$ & $(18,243)^{*}$ & $(17,048)^{*}$ & $(27,323)^{*}$ \\
\hline \multirow{2}{*}{\multicolumn{2}{|c|}{$\ln Y_{j}$}} & 0,371 & 0,630 & 0,569 & 1,368 \\
\hline & & $(13,694)^{*}$ & $(22,512)^{*}$ & $(19,827)^{*}$ & $(40,713)^{*}$ \\
\hline \multirow{2}{*}{\multicolumn{2}{|c|}{$\ln D_{i j}$}} & $-0,832$ & $-1,024$ & $-0,736$ & $-0,751$ \\
\hline & & $(-10,299)^{*}$ & $(-14,178)^{*}$ & $(-9,107)^{*}$ & $(-9,053)^{*}$ \\
\hline \multirow{2}{*}{\multicolumn{2}{|c|}{$A d j_{i j}$}} & 1,146 & 1,351 & 1,150 & 1,019 \\
\hline & & $(6,613)^{*}$ & $(7,514)^{*}$ & $(6,547)^{*}$ & $(6,511)^{*}$ \\
\hline \multirow{8}{*}{ 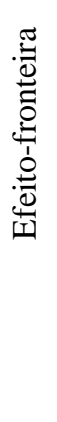 } & $E S T$ & 4,571 & & & 3,463 \\
\hline & (Intra-Estado) & $(11,681)^{*}$ & & & $(18,155)^{*}$ \\
\hline & $B R A$ & & 3,533 & & \\
\hline & (Internacional) & & & & \\
\hline & $D_{-}$Estados $^{3}$ & & & $\vdots$ & $\vdots$ \\
\hline & $\mathrm{F}$ & 494,242 & 571,765 & 110,902 & 111,309 \\
\hline & $\mathrm{R}^{2}$ Ajustado & 0,685 & 0,716 & 0,727 & 0,829 \\
\hline & $\mathrm{N}^{\mathrm{o}}$ Obs. & 1361 & 1361 & 1361 & 708 \\
\hline
\end{tabular}

Os valores entre parêntesis são as estatísticas t, de student; * indica significância a $1 \%$.

Em relação ao PIB dos estados exportadores, todos os coeficientes (elasticidades) encontrados apresentaram valor próximo da unidade, o que indica que aumentos no PIB do estado exportador levariam a aumentos

Os parâmetros estimados para as dummies de cada um dos estados e o DF estão apresentados na Figura1 e na Tabela A1, do apêndice. 
proporcionais no volume exportado. A elasticidade média do PIB dos estados importadores foi de 0,73 . O valor maior dessa mesma elasticidade na equação (4), igual a 1,368 , reflete a retirada das observações do comércio internacional daquela equação.

Todos os coeficientes encontrados para a variável distância apresentam significância estatística e, como esperado, têm sinais negativos, o que demonstra que o comércio é reduzido pelo aumento da distância entre os parceiros comerciais. A média encontrada para essa variável, nas quatro equações, foi de $-0,84$, o que mostra a redução de $8,4 \%$ no comércio para cada aumento de $10 \%$ nas distâncias. Esse valor é bem menor do que os encontrados por Daumar e Zignago (1,36 a 1,48), o que é justificado pela presença da variável adjacência, que capta parte da explicação para as exportações.

O coeficiente da variável dummy para adjacência apresentou valor médio igual a 1,17 e indica que o comércio entre estados vizinhos tende a ser, em média, 3,22 vezes ${ }^{4}$ maior do que com outros estados e países que não têm fronteira comum. Esse resultado está de acordo com os encontrados por Silva et al. (2007), os quais variaram de 3,2 a 3,7 para o comércio entre os estados adjacentes.

O efeito-fronteira, pela sua própria definição, é uma forma de medir o isolamento do comércio de uma região com as demais. Assim, quanto menor esse coeficiente, menor a diferença entre os "comércios" local e externo e maior a integração entre os parceiros.

Os coeficientes encontrados para a variável EST, na equação (4), foi igual a 3,46, o que indica que o comércio intra-estadual é, em média, 31,81 vezes maior que o interestadual. Quando se considera a inclusão do comércio internacional na análise (equação 1), o comércio intraestadual chega a ser 96 vezes maior do que com outros estados e países. Essa "preferência" pelo comércio local pode ser explicada, no Brasil, pelas diferenças em infra-estrutura física e social e pela cultura regional,

De acordo com Halversen e Palmquist (1980), em modelos na forma log-linear, a interpretação do coeficiente da variável dummy seria dada por e1,17 =3,22. 
já que as barreiras comerciais são mínimas entre os estados. O coeficiente estimado para a variável BRA, que mede o quanto o comércio interno (intra e inter-estado) é preferível ao internacional, equação (2), permite inferir que, em média, um estado brasileiro comercializa 34,23 vezes mais internamente do que com os parceiros internacionais. Esse resultado é conseqüência de todas as barreiras (comerciais ou não) existentes entre os estados brasileiros e os países considerados.

Na Figura 1 estão apresentadas as estimativas encontradas para o efeitofronteira no comércio intra-estadual para cada uma das UFs e o Distrito Federal. Quanto menor o valor do coeficiente estimado, menor a diferença do comércio local com o externo e, portanto, mais integrado é o estado com os demais e com os países (menor é a preferência pelo comércio local). Os valores apresentados na Figura 1 correspondem ao efeitofronteira interno (valor à esquerda da barra) e internacional (valor à direita), respectivamente.

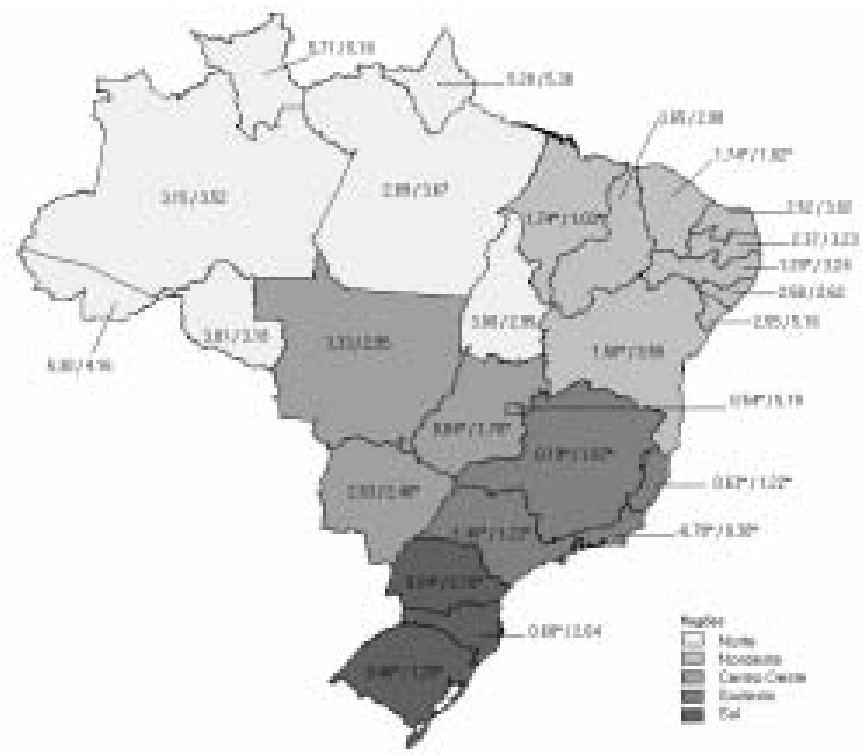

* Indica ausência de significância estatística.

Figura 1: Efeito-Fronteira para os Estados Brasileiros em 1999. 
As estimativas encontradas indicam que os estados das regiões Sudeste e Sul têm maior grau de abertura ao comércio tanto interno quanto internacional. Tal fato pode ser observado nos valores baixos para o efeito-fronteira apresentado por estados como São Paulo, Rio de Janeiro, Espírito Santo e Rio Grande do Sul. Todos esses estados têm importantes portos marítimos atuando como plataformas, no comércio para os demais estados. Os estados da região Nordeste apresentam "preferência" maior pelo comércio intra-estadual, como já mostrado por Hidalgo e Vergolino (1998) e por Silva et al. (2004). Os valores altos para o efeito-fronteira, na região Norte, justificam-se pelo fato de os estados ou regiões mais afastados apresentarem maiores "resistências" ao comércio e, por isso, tenderem a transacionar mais internamente.

\section{Conclusões}

Objetivou-se, neste estudo, mensurar e comparar o efeito-fronteira que há no comércio entre os estados brasileiros e seus principais parceiros comerciais internacionais, por meio de um modelo de gravidade. Foram utilizadas diferentes formas funcionais para captar o viés doméstico de comércio dos estados brasileiros, encontrando-se valores elevados para o efeito-fronteira, em todos os casos. Sua magnitude é função da assimetria econômica dos estados em relação aos seus parceiros comerciais internacionais; da seleção direcionada dos países importadores, desconsiderando os fluxos comerciais inexistentes (apenas 46 países); e, também, dos reflexos da desvalorização cambial ocorrida no ano de 1999, que, certamente, afetou os fluxos de comércio.

Com base nos resultados, observa-se que os estados brasileiros ainda estão pouco integrados entre si e ao mercado internacional. Há ainda grandes problemas de desigualdades produtivas e de renda no Brasil, que, aliados aos problemas de infra-estrutura física e de transportes entre os estados, contribuem significativamente para essa pequena integração de comércio. Dessa forma, poucos estados são responsáveis pela maior parcela das exportações e importações do país, principalmente os 
localizados nas regiões Sudeste e Sul, enquanto a grande maioria do comércio das UFs é realizado internamente.

Nesse sentido, a assimetria econômica e, por conseqüência, o baixo grau de integração comercial existente entre os estados deveriam ser corrigidos com políticas de desenvolvimento e de facilitação do comércio.

Sugere-se que, tão logo dados atuais sobre o comércio interestadual no Brasil estejam disponíveis, outros estudos sejam realizados, utilizando-se da análise de séries temporais para captar a evolução do efeito-fronteira dos estados brasileiros, e que sejam incluídas variáveis específicas, como a disponibilidade de infra-estrutura física, para indicar outros efeitos de resistência ao comércio.

\section{Referências}

AREASEG.COM - Distâncias entre as capitais brasileiras. < $\underline{\text { http:// }}$ www.areaseg.com/distancias.html>. (29/10/06)

AZEVEDO, André F. Z.; O Efeito do Mercosul sobre o Comércio: uma análise com o Modelo Gravitacional. Pesquisa e Planejamento Econômico. Vol. 34, n. 2, p. 307-339, 2004.

AZEVEDO, André F. Z.; PORTUGAL, M. S. e BARCELLOS NETO, P. C. F. Impactos comerciais da Área de Livre Comércio da Américas: uma aplicação do modelo gravitacional. Revista de Economia Contemporânea. Vol. 10, n. 2, p.237-267, 2006.

CASTRO, Newton; CARRIS, Larry; RODRIGUES, Bruno; Custos de transportes e a estrutura do comércio interestadual brasileiro. Pesquisa e Planejamento Econômico, v.29, n. 3, 1999.

CENTRO DE ESTUDOS PROSPECTIVOS E DE INFORMAÇÕES INTERNACIONAIS - CEPII. Databases - Distance. < http:// www.cepii.fr/anglaisgraph/bdd/distances.htm >. (04/11/06) 
DAUMAL, Marie; ZIGNAGO, Soledad; The Border Effects in Brazil. 2005. 〈http://www.dauphine.fr/globalisation/daumal2.pdf>. (15/02/07)

HALVERSEN, R.; PALMQUIST, R. The interpretation of dummy variables in semi logarithmic equations. American Economic Review. v.70, n.3, p. 474-475, 1980.

HELLIWELL, John F. Do national borders matter for Quebec's trade? Canadian Journal of Economics, v. 29, n. 3, p. 507-522, 1996.

HIDALGO, Álvaro B. e VERGOLINO, José R. O Nordeste e o comércio inter-regional e internacional: Um teste dos impactos por meio do modelo gravitacional. Economia Aplicada. Vol. 2 n.4, p. 707-725, 1998.

INSTITUTO BRASILEIRO DE GEOGRAFIA E ESTATÍSTICA IBGE. PIB por Unidade da Federação. 〈www.ibge.gov.br〉. (15/11/ 06)

McCALLUM, John. National borders matter: Canada-U.S. regional trade patterns. American Economic Review. Vol. 85, n. 3, p. 615-23, 1995.

MINISTÉRIO DO DESENVOLVIMENTO, INDÚSTRIA E COMÉRCIO EXTERIOR. Estatísticas da balança comercial dos estados. 〈http://www.desenvolvimento.gov.br>. (08/11/06)

MORAIS, Adriano G. Criação e desvio de comércio no Mercosul e Nafta. Faculdade de Economia e Administração, USP. Dissertação de Mestrado. 90p. 2005.

PENN WORLD TABLE. Database. $\lfloor$ http://pwt.econ.upenn.edu/>. (10/ $11 / 06)$

PIANI, Guida e KUME, Honório. Fluxos bilaterais de comércio e blocos regionais: uma aplicação do modelo gravitacional. Rio de Janeiro: Instituto de Pesquisas Econômicas Aplicadas - IPEA, 2000. 17p. (Texto para Discussão, n. 749). 
SÁ PORTO, P. C. Mercosul and regional development in Brazil: a gravity model approach. Estudos Econômicos, v. 32, n. 1, p. 125-153, 2002.

SILVA, Magno V. B.; JUSTO, Wellington R. e MAGALHÃES, André M. Comércio interestadual e internacional do Brasil e do Nordeste: Uma abordagem do modelo gravitacional. <www.bnb.gov.br/content/aplicacao/ ETENE/Anais/docs/2004-comercio-interestadual.pdf> (10/09/07)

SILVA, Orlando M.; ALMEIDA, Fernanda M.; OLIVEIRA, Bethania. M.; Intra-national versus international trade in Brazil: measuring the border effect. XII ANNUAL CONFERENCE: WESTERN HEMISPHERIC INTEGRATION IN A COMPETITIVE GLOBAL ENVIRONMENT, 2007, Texas and Monterrey, Anais..., Loredo: TAMIU, 2007.

TINBERGEN, Jan. Shaping the world economy: suggestions for an international economy policy. Twentieth Century Fund ,New York, 1962.

VASCONCELOS, José R.; OLIVEIRA, Márcio Augusto. Análise da matriz de fluxo do comércio interestadual no Brasil - 1999. Rio de Janeiro: IPEA, jul. 2006. (Texto para Discussão, 1159).

Abstract - Given that in Brazil there is a significant asymmetry in production and trade among states, this study had as objective to measure the border effect to the intranational and international trade, using it as an indicator of the degree of integration. Such effect measures the bias to the internal trade of a country or state, in comparison to the external trade. A gravity model, including different dummy variables was used as methodology for this study. Results showed that intra-state trade in Brazil was 32 and 96 times larger than interstate and international trade, respectively. Interstate trade, was shown to be around 34 times larger than international trade. The border effect calculated to each state trading to other states, and to the rest of the world, showed smaller values and larger commercial integration among the states of the southeast and south regions of Brazil.

Key words: International trade, border effect, gravity model. 


\section{Apêndice}

Tabela A1 - Estimativa dos Parâmetros Estaduais para o Comércio IntraEstadual e Internacional (MQO)

\begin{tabular}{|c|c|c|}
\hline \multirow{2}{*}{$\begin{array}{l}\text { Dummy } \\
\text { Estado }\end{array}$} & \multicolumn{2}{|c|}{ Equações } \\
\hline & Intra-Estado (4) & $\begin{array}{c}\text { Internacional } \\
(5)\end{array}$ \\
\hline \multirow{2}{*}{ D_AC } & 5,289 & 4,160 \\
\hline & $(4,418)^{\star * *}$ & $(2,780)^{\star \star *}$ \\
\hline \multirow{2}{*}{ D_AL } & 2,584 & 2,620 \\
\hline & $(2,152)^{\star \star}$ & $(1,729)^{*}$ \\
\hline \multirow{2}{*}{ D_AM } & 3,148 & 3,522 \\
\hline & $(2,667)^{\star * *}$ & $(2,354)^{\star \star}$ \\
\hline \multirow{2}{*}{ D_AP } & 5,283 & 5,384 \\
\hline & $(4,412)^{\star \star \star}$ & $(3,596)^{\star \star \star}$ \\
\hline \multirow{2}{*}{ D_BA } & 1,501 & 3,550 \\
\hline & $(1,269)^{\mathrm{ss}}$ & $(2,361)^{\star \star}$ \\
\hline \multirow{2}{*}{ D_CE } & 1,741 & 1,025 \\
\hline & $(1,466)^{\mathrm{s}}$ & $(0,681)^{\mathrm{s}}$ \\
\hline \multirow{2}{*}{ D_DF } & 0,544 & 5,187 \\
\hline & $(0,449)^{\mathrm{s}}$ & $(3,388)^{\star * *}$ \\
\hline \multirow{2}{*}{ D_ES } & 0,628 & 1,216 \\
\hline & $(0,525)^{\mathrm{s}}$ & $(0,803)^{\mathrm{s}}$ \\
\hline \multirow{2}{*}{ D_GO } & 0,842 & 1,692 \\
\hline & $(0,711)^{\mathrm{ns}}$ & $(1,128)^{\mathrm{ss}}$ \\
\hline \multirow{2}{*}{ D_MA } & 3,073 & 4,122 \\
\hline & $(2,592)^{\star * \star}$ & $(2,739)^{\star \star \star}$ \\
\hline \multirow{2}{*}{ D_MG } & 0,188 & 1,866 \\
\hline & $(0,159)^{\text {s }}$ & $(1,241)^{\mathrm{s}}$ \\
\hline \multirow{2}{*}{ D_MS } & 2,626 & 2,395 \\
\hline & $(2,216)^{\star *}$ & $(1,448)^{\mathrm{s}}$ \\
\hline \multirow{2}{*}{ D_MT } & 2,329 & 2,947 \\
\hline & $(1,971)^{\star *}$ & $(1,971)^{\star *}$ \\
\hline \multirow[t]{2}{*}{ D_PA } & 2,887 & 3,674 \\
\hline & $(2,445)^{* *}$ & $(2,467)^{* *}$ \\
\hline
\end{tabular}

\begin{tabular}{|c|c|c|}
\hline & Intra-Estado (4) & $\begin{array}{c}\text { Internacional } \\
\text { (5) }\end{array}$ \\
\hline \multirow{2}{*}{ D_PB } & 2,365 & 3,230 \\
\hline & $(1,979)^{* *}$ & $(2,140)^{* *}$ \\
\hline \multirow{2}{*}{ D_PE } & 1,202 & 3,240 \\
\hline & $(1,011)^{\mathrm{ss}}$ & $(2,142)^{\star \star}$ \\
\hline \multirow{2}{*}{ D_PI } & 3,654 & 2,982 \\
\hline & $(3,074)^{\star * *}$ & $(1,987)^{\star \star}$ \\
\hline \multirow{2}{*}{ D_PR } & 0,238 & 2,161 \\
\hline & $(0,200)^{\mathrm{s}}$ & $(1,435)^{\mathrm{s}}$ \\
\hline \multirow{2}{*}{ D_RJ } & $-0,775$ & 0,377 \\
\hline & $(-0,649)^{\mathrm{ps}}$ & $(0,248)^{\mathrm{s}}$ \\
\hline \multirow{2}{*}{ D_RN } & 2,524 & 3,024 \\
\hline & $(2,111)^{\star *}$ & $(2,001)^{\star *}$ \\
\hline \multirow{2}{*}{ D_RO } & 3,809 & 3,184 \\
\hline & $(3,204)^{\star \star \star}$ & $(2,132)^{\star \star}$ \\
\hline \multirow{2}{*}{ D_RR } & 5,713 & 5,180 \\
\hline & $(4,763)^{\star \star *}$ & $(3,451)^{* * *}$ \\
\hline \multirow{2}{*}{ D_RS } & 0,462 & 1,198 \\
\hline & $(0,390)^{\mathrm{ss}}$ & $(0,795)^{\mathrm{s}}$ \\
\hline \multirow{2}{*}{ D_SC } & 0,675 & 2,644 \\
\hline & $(0,567)^{\mathrm{ss}}$ & $(1,752)^{\star}$ \\
\hline \multirow{2}{*}{ D_SE } & 2,547 & 5,162 \\
\hline & $(2,116)^{\star *}$ & $(3,402)^{\star \star *}$ \\
\hline \multirow{2}{*}{ D_SP } & $-1,396$ & 1,231 \\
\hline & $(-1,172)^{\mathrm{s}}$ & $(0,814)^{\mathrm{ss}}$ \\
\hline \multirow{2}{*}{ D_TO } & 3,805 & 4,443 \\
\hline & $(3,192)^{\star \star \star}$ & $(2,961)^{\star \star \star}$ \\
\hline
\end{tabular}

Os valores entre parêntesis são as estatísticas t de student. *,** e ***, indicam significância nos níveis de 10 5 e $1 \%$, respectivamente, e ns indica ausência de significância. 
REVISTA DE ECONOMIA E AGRONEGÓCIO, VOL.5, $N^{o} 4$ 